\title{
O ensino de saúde e os currículos dos cursos de medicina veterinária: um estudo de caso
}

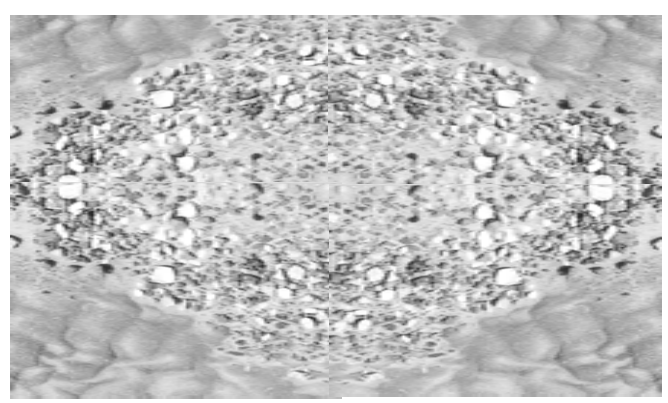

\author{
Márcia Regina Pfuetzenreiter ${ }^{1}$
} Arden Zylbersztajn²

PFUETZENREITER, M.R.; ZYLBERSZTAJN, A. Theaching of health and the curricula of schools of veterinary medicine: a case study, Interface - Comunic., Saúde, Educ., v.8, n.15, p.349-60, mar/ago 2004.

An investigation was conducted on the curricula of the pioneer schools of veterinary medicine in the country, as well as on the course offered by the University of the State of Santa Catarina, the analysis of documents having been the data collection procedure employed. Three main fields of action were identified in veterinary medicine, related with the practical activities carried out in the exercise of the profession and corresponding to the content of the courses: clinical veterinary practice, preventive veterinary medicine and public health, and animal husbandry and animal production. The connection between these three areas and the epistemological "style of thought" category proposed by L. Fleck was articulated. The analysis of the data obtained indicated that social and preventive aspects were given scanty attention in veterinary medicine courses, causing the "style of thought" that focuses on preventive veterinary medicine and public health to be given little emphasis in comparison with the other "styles of thought" found in the profession.

KEY WORDS: Veterinary Medicine; curriculum; veterinary education; health education; "style of thought.

Realizou-se uma investigação sobre os currículos dos cursos de medicina veterinária pioneiros no país e do curso da Universidade do Estado de Santa Catarina, utilizando a análise de documentos como procedimento de coleta de dados. Identificaram-se três principais campos de atuação dentro da medicina veterinária, relacionados com as atividades práticas realizadas na profissão e que correspondem aos conteúdos curriculares dos cursos: clínica veterinária, medicina veterinária preventiva e saúde pública, e zootecnia e produção animal. Foi articulada a relação entre essas áreas e a categoria epistemológica "estilo de pensamento" proposta por L. Fleck. A análise dos dados obtidos indicou que as concepções de natureza social e preventiva recebem pouco destaque dentro dos cursos de Medicina Veterinária, o que faz com que o estilo de pensamento da Medicina Veterinária Preventiva e Saúde Pública seja pouco enfatizado em relação aos outros estilos de pensamento presentes na profissão.

PALAVRAS-CHAVE: Medicina Veterinária; currículo, educação veterinária; ensino em saúde; "estilo de pensamento".

${ }_{1}^{1}$ Professora Assistente Doutor, Departamento de Medicina Veterinária Preventiva e Tecnologia, Universidade do Estado de Santa
Catarina, Centro de Ciências Agroveterinárias/UDESC. <marcia@cav.udesc.br>

${ }^{2}$ Professor Assistente Doutor, Departamento de Física, Universidade Federal de Santa Catarina/UFSC. <arden@fsc.ufsc.br>

\footnotetext{
${ }^{1}$ Av. Luiz de Camões, 2090

Lages, SC

88.530-000
} 


\section{Introdução}

A saúde pública veterinária é resultado da aplicação do conhecimento profissional do médico veterinário para a proteção e promoção da saúde humana e também para a economia. Esta atividade reflete os interesses comuns e indica oportunidades de interações proveitosas entre as medicinas veterinária e humana. Pela utilização dos conhecimentos biomédicos básicos e pela natureza de profissão cruzada, o sanitarista veterinário realiza uma função única na equipe de saúde pública, sendo difícil separar as atividades de saúde pública veterinária da saúde humana (Bögel, 1992; Boletim de la Oficina Sanitaria Panamericana, 1992).

Ainda que a medicina veterinária tenha um papel fundamental a desempenhar no campo da saúde pública havendo, portanto, necessidade de profissionais veterinários especializados nesta área, alguns estudos (Arámbulo E Ruíz, 1992; Boletim de la Oficina Sanitaria Panamericana, 1992; Palermo Neto, 1995) ressaltam que as escolas de veterinária não têm enfatizado a capacitação nesse setor. Atualmente, mesmo constando dos currículos dos cursos, não há uma orientação significativa para a medicina veterinária preventiva e saúde pública.

A fundamentação teórica deste trabalho está baseada na epistemologia de L. Fleck (1986), que condiciona os conhecimentos a fatores históricos, psicológicos e sociais e segundo o qual os conceitos estão ligados ao desenvolvimento histórico de algumas linhas coletivas de pensamento. $\mathrm{O}$ autor baseou a categoria denominada de "estilo de pensamento" essencialmente na atividade prática. Este tema foi discutido em vários de seus textos relacionados a aspectos sociais como a utilização de instrumentos por determinado coletivo, com o emprego de uma linguagem própria, com o ensino e com a percepção direcionada para a aquisição de habilidades, prática $e$ experiência pelos indivíduos para tomarem parte de um grupo.

Para as finalidades da investigação, foi estabelecido um paralelo entre as práticas desenvolvidas em medicina veterinária $e$ as categorias epistemológicas estabelecidas por Fleck. Assim, as atividades práticas exercidas pelos diversos segmentos da profissão foram adaptadas ao sistema de pensamento do epistemólogo. Com base em Rista \& Bastos Santos (2001), Rosenberg \& Olascoaga (1991) e Brasil (2003), que classificam as grandes áreas de atuação do médico veterinário, este trabalho propõe a seguinte disposição dos grupos de atuação para a profissão:

a) Clínica Veterinária ( $\mathrm{CV}$ ): inclui conhecimentos ligados às atividades de clínica, cirurgia, patologia e fisiopatologia da reprodução.

b) Zootecnia e Produção Animal (ZPA): incorpora conteúdos relacionados aos sistemas de criação, manejo, nutrição, biotécnicas da reprodução, exploração econômica e ecologicamente sustentável.

c) Medicina Veterinária Preventiva e Saúde Pública (MVPSP): envolve os conteúdos relacionados a planejamento, administração e educação em saúde, epidemiologia, zoonoses, ecologia e proteção ao meio ambiente e inspeção higiênica e sanitária dos produtos de origem animal.

As grandes áreas de atuação representam os pilares fundamentais do currículo dos cursos de medicina veterinária que estão assentados sobre os princípios das ciências básicas. Cada um desses três agrupamentos se constitui 
${ }^{3}$ A Comissão Nacional de Ensino de Medicina Veterinária (Conselho Federal de Medicina Veterinária, 1992)

considera como pioneiros os primeiros cursos de medicina veterinária fundados no país e que mantiveram seu funcionamento: Universidade Federal Rural do Rio de Janeiro (UFRRJ); Universidade Federal do Rio Grande do Sul (UFRGS);

Universidade Federal do Paraná (UFPR)

Universidade Federal de Minas Gerais (UFMG); Universidade de São Paulo (USP):

Universidade Federal Fluminense (UFF); Universidade Federal Rural de Pernambuco (UFRPE); Universidade Federal da Bahia (UFBA).

${ }^{4}$ As matérias básicas constituem uma form de pensamento própria compartilhada com os cursos da área das ciências da saúde. em grandes áreas que se refletem na atuação profissional veterinária, reúnem grupos de pessoas que compartilham a mesma linguagem, os mesmos conceitos, instrumentos de trabalho e procedimentos, formando modos de pensar distintos.

O presente trabalho apresenta a análise dos resultados da investigação sobre a forma como os conteúdos ligados ao estilo de pensamento da medicina veterinária preventiva e saúde pública estão inseridos nos cursos de medicina veterinária pioneiros no país e de uma comparação com o curso de medicina veterinária da Universidade do Estado de Santa Catarina.

\section{Material e métodos}

Foram analisadas as grades curriculares dos cursos de medicina veterinária das escolas pioneiras de medicina veterinária ${ }^{3} e$ do curso da Universidade do Estado de Santa Catarina (UDESC). Os currículos estudados foram aqueles fornecidos pelas instituições ou disponibilizados pelos cursos na internet durante o ano de 2003.

As categorias de análise utilizadas foram os estilos de pensamento presentes dentro da medicina veterinária, correspondentes aos campos de atuação da profissão: clínica veterinária, medicina veterinária preventiva $e$ saúde pública e zootecnia e produção animal. Os conteúdos das disciplinas obrigatórias de cada curso foram verificados e classificados dentro dos estilos de pensamento, observando a carga horária destinada a cada estilo dentro do curso ${ }^{4}$. Os programas curriculares das disciplinas ligadas à medicina veterinária preventiva e saúde pública foram examinados. Com base nas informações obtidas, foi estabelecida uma comparação entre os currículos com o propósito de analisar o ensino da medicina veterinária preventiva $e$ saúde pública.

\section{Resultados} (UFBA)

Curso de medicina veterinária da Universidade Federal da Bahia

O curso de medicina veterinária da UFBA tem carga horária total de 3.630 horas, com duração média de cinco anos e máxima de oito anos. Há obrigatoriedade da realização de um estágio supervisionado de 360 horas. A distribuição das disciplinas dentro dos estilos de pensamento está descrita na tabela 1, que mostra que o estilo de pensamento de Clínica Veterinária é o que concentra a maior carga horária, com $34,55 \%$.

A observação dos programas das disciplinas pertencentes ao estilo de pensamento da medicina veterinária preventiva e saúde pública revelou haver pouca ênfase para a área de administração e planejamento em saúde animal e saúde pública. Os conteúdos relacionados à educação e saúde não são citados. $O$ estilo da medicina veterinária preventiva e saúde pública apresenta $60 \%$ de sua carga horária voltada para as disciplinas de inspeção e tecnologia e nenhuma disciplina aborda conteúdos relacionados ao meio ambiente. A tabela 2 mostra a distribuição de carga horária relativa aos conteúdos de medicina veterinária preventiva e saúde pública para todos os cursos analisados. 
PFUETZENREITER, M.R.; ZYLBERSZTAJN, A.

Curso de medicina veterinária da Universidade Federal Fluminense (UFF)

O curso da Faculdade de Medicina Veterinária da UFF tem uma duração mínima de oito e máxima de 16 semestres. A carga horária total é de 4650 horas, sendo distribuída em 4110 horas de disciplinas obrigatórias, 450 horas de estágio obrigatório, sessenta horas de disciplinas optativas e trinta de disciplinas eletivas.

A clínica veterinária ocupa a maior parte da carga horária do curso, seguida de perto pelas disciplinas básicas (tabela 1). A disciplina de zoonoses está localizada no oitavo período do curso e versa sobre transmissão e prevenção de doenças, sem que os alunos tenham elementos prévios de epidemiologia básica. O programa, muito extenso, comprimido em uma carga horária pequena, faz com que a disciplina de medicina veterinária preventiva e saúde pública só forneça noções muito genéricas e superficiais aos estudantes, sem contribuir para a formação de um pensamento preventivo e populacional. Dentro desta disciplina, conteúdos como administração e planejamento em saúde e de educação em saúde são muito pouco enfatizados. Chama a atenção o excessivo número de disciplinas e a carga horária dedicada à inspeção e tecnologia de produtos, com um detalhamento que vai além do nível de graduação (tabela 2).

\section{Curso de medicina veterinária da Universidade Federal de Minas Gerais (UFMG)}

O tempo previsto para integralização curricular do curso da escola de medicina veterinária da UFMG é de dez semestres, com um mínimo de oito e máximo de 17 semestres. A grade curricular conta com uma disciplina de sociologia e antropologia, importante para fornecer ao aluno uma visão mais voltada para a sociedade, fortalecendo a compreensão da importância do coletivo, que auxilia o estabelecimento do estilo de pensamento da medicina veterinária preventiva e saúde pública. É o currículo que apresenta a maior percentagem da carga horária destinada a esta área de atuação, com 13,89\% (tabela 1).

A disciplinas do estilo de pensamento de medicina veterinária preventiva e saúde pública ligadas à inspeção e tecnologia de produtos de origem animal ocupam mais da metade da carga horária desse estilo (tabela 2). $\mathrm{O}$ programa da disciplina de planificação em saúde animal, como a própria denominação anuncia, trata apenas o tema sob o ponto de vista da medicina veterinária, sem uma abordagem mais ampla que envolva a saúde pública. Esta lacuna dificulta a preparação do profissional para atuar em equipes multidisciplinares de saúde, no desenvolvimento de tarefas que extrapolem o âmbito da saúde animal e envolvam também a saúde pública.

\section{Curso de medicina veterinária da Universidade Federal do Paraná (UFPR) \\ $O$ curso de medicina veterinária da UFPR tem duração média de cinco anos, podendo variar entre quatro e oito anos. A carga horária total é de 4500 horas, sendo 3795 horas de disciplinas de formação básica $e$ profissionalizante, 315 de estágio curricular obrigatório, 240 de disciplinas}


optativas e 150 de atividades complementares. Destas, vinte deverão ser cumpridas na área de ciências humanas e sociais.

Na tabela 1 observa-se que o estilo de pensamento de clínica veterinária se sobrepõe aos demais em termos de carga horária, com 35,18\%.

Este currículo está em fase de implantação, tendo sido adotado no ano letivo de 2002. Um ponto curioso é a obrigatoriedade para o aluno em cursar pelo menos vinte horas em atividades complementares na área de ciências humanas e sociais. Entretanto, seria mais interessante se estes conteúdos estivessem integrados aos demais conteúdos do curso, para que o aluno pudesse perceber a área dentro da profissão.

As disciplinas ligadas à inspeção e tecnologia de alimentos ocupam quase metade da carga horária total do estilo de pensamento da medicina veterinária preventiva e saúde pública (tabela 2). Ganham destaque as disciplinas relacionadas ao meio ambiente que, apesar de terem pequena representatividade dentro do estilo de pensamento em termos de carga horária, abordam importantes temas ligados à saúde.

\section{Curso de medicina veterinária da Universidade Federal do Rio} Grande do Sul (UFRGS)

O currículo do curso de medicina veterinária da UFRGS está estruturado em 11 semestres e tem uma carga horária total de 5.070 horas (4470 horas em disciplinas obrigatórias e seiscentas horas em estágio curricular). Está constituído por quatro linhas curriculares que configuram os seguintes campos de conhecimentos: patologia animal, zootecnia, saúde pública, e ciências sociais. Pela representação dos estilos de pensamento nas disciplinas obrigatórias do curso (tabela 1), pode-se observar que o estilo de clínica veterinária retém a maior carga horária, com 42,62\%.

No que diz respeito especificamente ao estilo da medicina veterinária preventiva e saúde pública, existe uma interação entre as disciplinas e o cuidado em preparar o estudante para os conteúdos que serão vistos posteriormente. Chama a atenção a disciplina de ecologia aplicada à medicina veterinária que trata de temas como a ecologia médica $e$ oportuniza aos alunos a aquisição de uma visão voltada para a saúde dos animais $e$, conseqüentemente, para a melhoria da saúde humana $e$ da qualidade de vida. Como ela é ministrada nas primeiras fases do curso, fornece subsídios para as outras disciplinas desenvolverem e fortalecerem um pensamento preventivo e social. Outras disciplinas como a medicina veterinária preventiva e a veterinária em saúde pública enfatizam a promoção da saúde dando um enfoque populacional e preventivo, além de trazerem elementos das ciências sociais aplicadas à saúde. Mais da metade da

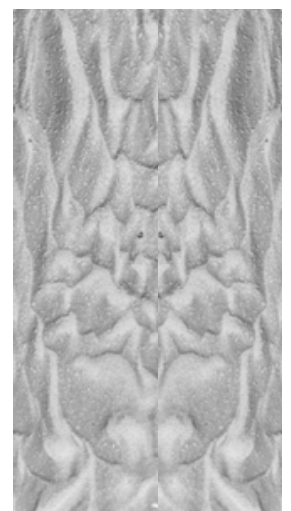
carga horária destinada ao estilo de pensamento se concentra nas disciplinas de inspeção e tecnologia de alimentos.

Curso de medicina veterinária da Universidade Federal Rural de Pernambuco (UFRPE)

O curso da UFRPE tem a duração de 11 semestres, com uma carga horária de 4140 horas, incluindo o estágio supervisionado obrigatório, com 360 horas, e mais trinta horas referentes à prática de educação física. 
PFUETZENREITER, M.R.; ZYLBERSZTAJN, A.

Este currículo apresenta uma das maiores cargas horárias para o estilo de pensamento da medicina veterinária preventiva e saúde pública, com $13,2 \%$ do total do curso (tabela 1). Chama a atenção a oferta de uma disciplina - a filosofia da ciência e ética - na área de filosofia e epistemologia. É um dos poucos currículos que se preocupa explicitamente em fornecer ao aluno conhecimentos voltados para este campo e que são importantes para que ele tenha uma noção mais ampla sobre a natureza da ciência.

Mais de $60 \%$ da carga horária das disciplinas ligadas ao estilo de pensamento de medicina veterinária preventiva e saúde pública é dedicada à inspeção e tecnologia de alimentos (tabela 2). Somente no nono semestre do curso, na disciplina de higiene veterinária e saúde pública, são trabalhadas as concepções de saúde e doença - quando o aluno já teve contato com outros conteúdos de patologia. O que pode ocorrer é que o estudante não teve oportunidade de apreender de maneira correta essas concepções e pode formar noções equivocadas. Neste curso ocorre o mesmo que na Universidade Federal de Minas Gerais, em que apenas é dado enfoque para o planejamento em saúde animal, sem preparar os alunos para efetuarem ações dentro da saúde pública.

Curso de medicina veterinária da Universidade Federal Rural do Rio de Janeiro (UFRRJ)

O curso de medicina veterinária da UFRRJ tem a duração mínima de cinco anos e máxima de oito. A carga horária total do curso é de 4245 horas, sendo $4185 \mathrm{em}$ disciplinas obrigatórias e sessenta horas em optativas, não sendo exigido estágio curricular.

Este curso é o que apresenta a maior carga horária destinada ao estilo de pensamento da clínica veterinária, com 42,65\% (tabela 1). Há uma grande vantagem de carga horária para as disciplinas do segmento de inspeção e tecnologia de alimentos dentro da medicina veterinária preventiva e saúde pública. Mais de $70 \%$ da carga horária desse estilo de pensamento é dedicada às disciplinas relacionadas à Inspeção, em detrimento de outras sub-áreas dentro do estilo (tabela 2). Há somente uma disciplina que aborda conteúdos relacionados à epidemiologia e zoonoses (higiene e saúde pública) que, pelo pouco tempo disponível, aborda esses temas de forma superficial. A parte de administração e planejamento em saúde e também de educação em saúde não são mencionadas no programa dessa disciplina. Como conseqüência, pode-se especular que o aluno sentirá dificuldades para adotar um pensamento preventivo e social em sua prática profissional.

\section{Curso de medicina veterinária da Universidade de São Paulo (USP)}

Além da carga horária de 4485 horas em disciplinas obrigatórias, são adicionadas trinta horas em disciplinas optativas e 480 horas de estágio ao currículo da faculdade de medicina veterinária e zootecnia da USP, que tem uma duração ideal de dez semestres, com máximo de 18. O curso apresenta uma percentagem grande de sua carga horária destinada ao estilo de pensamento da clínica veterinária (42,47\%), ao mesmo tempo em que o estilo da medicina veterinária preventiva e saúde pública recebe uma 
percentagem bastante baixa $(9,7 \%)$ - tabela 1 .

Dentro do estilo de pensamento da medicina veterinária preventiva $e$ saúde pública, não há disciplinas específicas ligadas ao meio ambiente (tabela 2). Apesar da pequena carga horária destinada a esse estilo de pensamento, um ponto positivo observado neste currículo é que a epidemiologia começa a ser vista na metade do curso, auxiliando a formação do pensamento preventivo e populacional. A disciplina de gerenciamento em saúde animal e saúde pública enfatiza assuntos pouco tratados nas disciplinas do segmento de saúde, mas importantes para consolidar a visão social, tratando não somente da planificação em programas de saúde animal, mas também de saúde pública.

Curso de medicina veterinária da Universidade do Estado de Santa Catarina (UDESC)

O curso de medicina veterinária da UDESC está localizado na cidade de Lages e tem duração de dez semestres, apresentando uma carga horária total de 4890 horas (4170 de disciplinas obrigatórias, 120 de disciplinas optativas e seiscentas para o estágio profissional obrigatório).

O estilo de pensamento da medicina veterinária preventiva e saúde pública ainda se apresenta de maneira incipiente dentro do currículo, e a quantidade de tempo dedicada aos conteúdos pertinentes à saúde pública é muito mais baixa do que para os estilos de clínica veterinária e de zootecnia e produção animal, sendo inclusive inferior às matérias básicas e aos outros estilos de pensamento (tabela 1).

Os estudantes começam a tomar contato com o estilo de pensamento da medicina veterinária preventiva e saúde pública somente a partir do quarto semestre do curso, e de maneira muito superficial. Nesta fase, a maior carga horária está concentrada sobre o estilo da clínica veterinária e também sobre as matérias básicas. Somente no final do curso - a partir do $8^{\circ}$ semestre - é que o estilo de pensamento da medicina veterinária preventiva e saúde pública começa realmente a ser apresentado para os alunos, quando todos os outros estilos já tiveram oportunidade de se desenvolver e de despertar o interesse dos estudantes. Isto faz com que o pensamento preventivo $e$ populacional tenha sérias dificuldades para se instalar, confome Pfuetzenreiter (2003) constatou a partir de entrevistas com estudantes do curso.

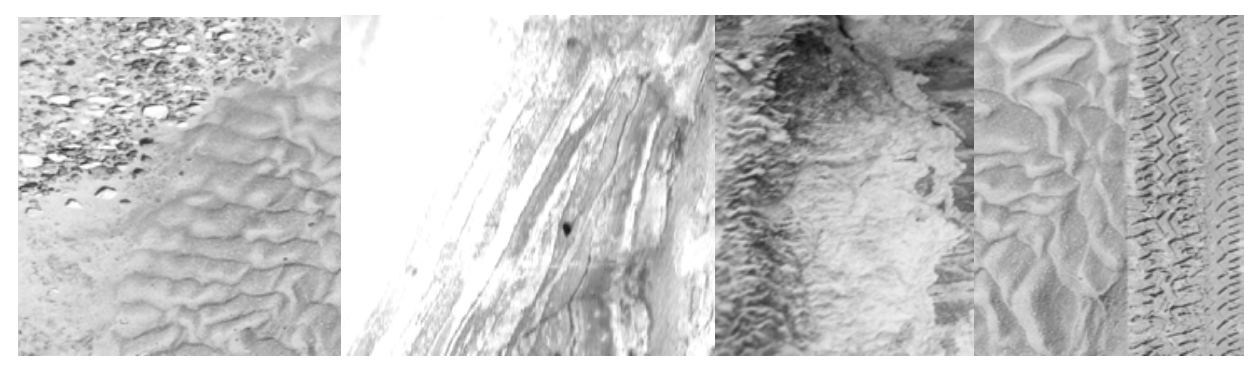


PFUETZENREITER, M.R.; ZYLBERSZTAJN, A.

Tabela 1 - Percentagem de carga horária referente aos estilos de pensamento nas disciplinas obrigatórias dos currículos dos cursos de medicina veterinária das universidades pioneiras do Brasil

\begin{tabular}{|c|c|c|c|c|c|c|}
\hline \multirow{3}{*}{ CURSO } & \multicolumn{6}{|c|}{ ESTILODE PENSAMENTO } \\
\hline & $\mathrm{CV}^{1}$ & MVPSP $^{2}$ & $\mathrm{ZPA}^{3}$ & $\mathrm{MB}^{4}$ & Outros $^{5}$ & $\mathrm{CH}$ Total $^{6}$ \\
\hline & $\%$ & $\%$ & $\%$ & $\%$ & $\%$ & $\%$ \\
\hline UFBA & 34,55 & 10,47 & 23,04 & 26,18 & 5,76 & 3630 \\
\hline UFF & 35,04 & 12,77 & 14,23 & 31,75 & 6,20 & 4650 \\
\hline UFMG & 38,49 & 13,89 & 16,27 & 25,79 & 5,56 & 3870 \\
\hline UFPR & 35,18 & 13,04 & 19,76 & 31,23 & 0,79 & 4500 \\
\hline UFRGS & 42,62 & 10,06 & 21,48 & 21,14 & 4,70 & 5070 \\
\hline UFRPE & 37,20 & 13,20 & 19,60 & 24,40 & 5,60 & 4140 \\
\hline UFRRJ & 42,65 & 10,05 & 14,69 & 28,31 & 4,30 & 4245 \\
\hline USP & 42,47 & 9,70 & 15,38 & 30,77 & 1,67 & 4995 \\
\hline UDESC & 41,73 & 5,75 & 17,27 & 29,86 & 5,39 & 4890 \\
\hline Média dos cursos pioneiros & 38,62 & 11,64 & 17,96 & 27,42 & 4,36 & 4388 \\
\hline Média de todos os cursos & 38,96 & 10,98 & 17,88 & 27,69 & 4,48 & 4443 \\
\hline
\end{tabular}

Notas:

1 Estilo de pensamento de clínica veterinária.

2 Estilo de pensamento de medicina veterinária preventiva e saúde pública.

3 Estilo de pensamento de zootecnia e produção animal.

$4 \mathrm{MB}$ : matérias básicas.

5 Outros/Mesclas: outros estilos de pensamento e mesclas de estilos.

$6 \mathrm{CH}$ Total: carga horária total do curso, que inclui as disciplinas e o estágio obrigatório.

Tabela 2 - Carga horária relativa às atividades da medicina veterinária preventiva e saúde pública nos cursos de medicina veterinária das universidades pioneiras do Brasil

\begin{tabular}{|c|c|c|c|c|}
\hline CURSO & $\begin{array}{c}\text { INSPEÇÃOE } \\
\text { TECNOLOGIA } \\
(\%)\end{array}$ & $\begin{array}{c}\text { MEIO } \\
\text { AMBIENTE }^{2} \\
(\%)\end{array}$ & $\begin{array}{c}\text { SAÚDE }^{3} \\
(\%)\end{array}$ & $\begin{array}{c}\text { MEIOAMBIENTE } \\
\text { E SAÚDE } \\
(\%)\end{array}$ \\
\hline UFBA & 60,00 & - & 40,00 & - \\
\hline UFF & 74,29 & 8,57 & 17,14 & - \\
\hline UFMG & 62,86 & 8,57 & 28,57 & - \\
\hline UFPR & 48,48 & 9,09 & 36,36 & 6,06 \\
\hline UFRGS & 53,33 & 6,67 & 40,00 & - \\
\hline UFRPE & 63,64 & 12,12 & 24,24 & - \\
\hline UFRRJ & 71,43 & 14,28 & 14,28 & - \\
\hline USP & 44,83 & - & 55,17 & - \\
\hline UDESC & 42,50 & 12,5 & 25,00 & - \\
\hline Média dos cursos pioneiros & 59,86 & 7,41 & 31,97 & - \\
\hline Média de todos os cursos & 60,15 & 7,98 & 31,20 & - \\
\hline
\end{tabular}

Notas:

1 Disciplinas com conteúdos relacionados à inspeção e tecnologia de produtos de origem animal.

2 Disciplinas com conteúdos relacionados à ecologia e meio ambiente.

3 Disciplinas com conteúdos relacionados à epidemiologia, zoonoses, educação em saúde; administração e planejamento em saúde. 
${ }^{5}$ A concepção biologicista se fundamenta em uma visão reducionista enfatizando a doença $e$ $o$ atendimento individual. A visão higienista preventivista se sustenta na multicausalidade e concentra sua prática na prevenção e higiene. Finalmente, a atitude médico-social entende a saúde enquanto processo determinado socialmente e concentra sua atenção na coletividade.

\section{Discussão}

O curso de medicina veterinária da UDESC está entre os cursos com maior carga horária (4890 horas), sendo inferior apenas à UFRGS (5.070 horas) e à USP (4995 horas). As escolas de educação superior com menor carga horária pertencem à UFMG, com 3870 horas, e a UFBA, com 3630 horas. Entretanto, não há exigência de estágio curricular obrigatório para o curso da UFMG.

Em todos os cursos é observada maior representatividade para o estilo de pensamento de clínica veterinária, seguida pelo estilo da zootecnia $e$ produção animal, com a menor participação para a medicina veterinária preventiva e saúde pública (tabela 1). A pequena carga horária para o campo da medicina veterinária preventiva e saúde pública nos cursos já foi observada no passado por Cruz \& Acha (1972).A baixa representatividade deste último estilo indica que ele ainda não se firmou dentro da estrutura dos cursos, apesar da grande importância dos conhecimentos que traz para a atividade profissional nas diferentes áreas de atuação profissional. A situação apresentada no curso da UDESC é ainda mais crítica, dado que a percentagem de carga horária destinada ao estilo da medicina veterinária preventiva e saúde pública representa menos da metade da média apresentada pelos demais cursos analisados.

Cutolo (2001) mostra que há três concepções de saúde - a preventivista, a médico-social e a biologicista - nos cursos de graduação em medicina, que seriam elementos caracterizadores dos estilos de pensamento ${ }^{5}$. No curso estudado pelo autor, a carga horária destinada às disciplinas que oferecem uma visão reducionista é muito superior em comparação com as atividades voltadas à área de ciências humanas. No modelo de ensino criticado pelo autor, essa visão é dominante e, apesar de hegemônica, não é a única $e$ convive com as demais. Sobre o aprendizado dentro de determinado estilo, Fleck (1986) explica que o ver formativo direto e desenvolvido exige o desenvolvimento da capacidade para adquirir uma visão direcionada para determinada perspectiva, reduzindo drasticamente a habilidade para outras formas de percepção.

Nas descrições das características e objetivos dos cursos e dos campos de atuação na profissão, os estabelecimentos de ensino estudados, inclusive o curso da UDESC, colocam em destaque a medicina veterinária preventiva $e$ saúde pública. Entretanto, pela análise efetuada, na prática as outras áreas se sobressaem muito mais, mesmo naqueles cursos que estão localizados nos centros de ciências médicas e da saúde de suas instituições.

Em todas as escolas superiores estudadas os conteúdos do estilo de pensamento da medicina veterinária preventiva e saúde pública estão desarticulados dos outros estilos, sem inter-relação com os demais conhecimentos da profissão médico-veterinária. Mesmo dentro do próprio estilo de pensamento há necessidade de haver maior conexão entre os conteúdos das disciplinas. Alguns autores (Arámbulo \& Ruíz, 1992; Palermo Neto, 1995) sugerem a mudança de perspectiva dos currículos dos cursos de medicina veterinária para uma formação mais voltada para os aspectos de saúde pública.

As cargas horárias dos conteúdos relativos às diversas atividades dentro 
do estilo de pensamento da medicina veterinária preventiva e saúde pública estão distribuídas sem uniformidade nas disciplinas da área (tabela 2). As disciplinas que tratam de conteúdos de inspeção e tecnologia de alimentos que apresentam uma visão higienista-preventivista - se destacam sobre as demais, enquanto as disciplinas que trabalham conteúdos relacionados ao meio ambiente - caracterizados pela visão social - são as que apresentam menor carga horária. Os conteúdos ligados à epidemiologia, zoonoses, educação em saúde, administração e planejamento em saúde - que possuem tendências de natureza higienista-preventivista e também social - são os que possuem maior irregularidade em sua distribuição nos cursos, sinal de que não estão ainda bem firmados dentro do estilo de pensamento da medicina veterinária preventiva e saúde pública.

Uma observação da bibliografia utilizada nas disciplinas do estilo de pensamento da medicina veterinária preventiva e saúde pública revela que há um pequeno número de referências relacionadas às ciências sociais aplicadas à saúde e quase ausência na área de educação em saúde. Este fato indica que há necessidade de uma atenção maior para a saúde coletiva, para que os alunos passem a ter uma compreensão completa da saúde das populações levando em consideração os aspectos sociais, culturais e econômicos e passem a ter um grau maior de comprometimento com a saúde da população. A pequena aproximação da medicina veterinária preventiva e saúde pública com as ciências humanas e sociais e com a educação em saúde é um reflexo da falta de interação dos cursos com outras áreas do conhecimento.

Um outro ponto que chama a atenção é que os alunos tomam contato com certos conteúdos de medicina veterinária preventiva e saúde pública em períodos muito adiantados do curso. Um exemplo é a compreensão dos modelos de saúde e doença. Este assunto está inserido da metade para o final do curso, quando os alunos já tiveram outros conhecimentos que trataram especificamente do patológico, sem versar sobre a saúde, fazendo com que eles apresentem concepções próprias sobre o fenômeno, distantes dos conceitos estabelecidos pela saúde coletiva. Como um exemplo desta constatação, os formandos do curso de medicina veterinária da UDESC possuem uma representação de saúde e doença como aspectos antagônicos, quando deveriam compreender o fenômeno de forma mais ampla (Pfuetzenreiter et al., 2001).

Percebe-se que certas atitudes dos coletivos de pensamento tendem a se perpetuar, caracterizando uma forte resistência a mudanças. Fleck (1986) alerta que existe uma tendência à persistência das idéias e quem não concorda com a estrutura organizada é tratado como exceção. Pelo comportamento adotado pelos coletivos de pensamento nos cursos observa-se que cada estilo de pensamento vai se tornando uma estrutura cada vez mais rígida e que deixa pouco espaço para o desenvolvimento de outras formas de pensamento (Pfuetzenreiter, 2003).

\section{Conclusões}

Todos os currículos analisados seguem um modelo de estrutura curricular fixa, seguindo os parâmetros propostos pelo currículo mínimo de 1984, que favorece a compartimentalização das disciplinas, sem haver inter-relação de umas com as outras. Como conseqüência, percebe-se uma tendência de 
isolamento das mesmas, dos conteúdos e, conseqüentemente, das formas de pensamento dentro do curso, refletindo-se em uma forma de ensino fragmentado e desprovido de um sentido mais amplo para o aluno.

Pelo exposto, concluímos que o foco principal das escolas estudadas é para uma formação tecnicista baseada no desenvolvimento de conhecimentos teóricos e práticos específicos das áreas de atuação da medicina veterinária, sem uma preocupação explícita com uma formação mais ampla, que contemple outros domínios do conhecimento humano, como as ciências humanas $e$ sociais - aspectos fundamentais que auxiliam no estabelecimento de um pensamento voltado para a medicina veterinária preventiva e saúde pública.

Considerando o quadro descrito sugerimos que, na implantação $e$ implementação das recentes Diretrizes Curriculares para os cursos de medicina veterinária (Brasil, 2003), se busque estabelecer a identidade da medicina veterinária preventiva e saúde pública e que sejam traçados os objetivos do ensino desse estilo de pensamento. Frente ao ritmo de crescimento dos conhecimentos na área, torna-se importante ainda que sejam oferecidas aos estudantes oportunidades que contemplem o desenvolvimento da capacidade de pensar, elaborar juízos e de articulação com os conteúdos dos demais estilos.

Propomos que um plano de ensino para a medicina veterinária preventiva $e$ saúde pública deve proporcionar aos estudantes a aquisição de conhecimentos $e$ prepará-los por meio de experiências de aprendizado para solucionarem os problemas de comunidades. A elaboração do saber aliada à pesquisa e à extensão universitária permeariam a construção curricular da área de medicina veterinária preventiva e saúde pública (e também das demais áreas do curso de medicina veterinária).

Sugerimos, também, que a conscientização dos profissionais de saúde e da população em geral sobre o papel do médico veterinário para a saúde pública, principalmente em relação ao controle de zoonoses seja estimulada, com os cursos se preocupando em fornecer uma compreensão maior sobre a área, para facilitar as discussões de caráter interdisciplinar e promover a colaboração entre os profissionais envolvidos no tema.

Finalmente, destacamos a importância de que a implementação do estilo de pensamento da medicina veterinária preventiva e saúde pública nos currículos atinja todas as esferas de ação deste estilo como os conteúdos relacionados à ecologia e meio ambiente, epidemiologia, zoonoses, educação em saúde, administração e planejamento em saúde e à inspeção e tecnologia de produtos de origem animal.

\section{Referências}

ARÁMBULO, P. III; RUÍZ, A. Situación actual y futura de la medicina veterinaria. Educ. Méd. Salud, v.26 n.2, p.263-76, 1992.

BÖGEL, K. Veterinary public health perspectives: trend assessment and recommendations. Rev. Sci. Tech., v.11, n.1, p.219-39, 1992.

BOLETIM DE LA OFICINA SANITARIA PANAMERICANA. La salud pública veterinaria, v.113, n.5-6, p.494501, 1992.

BRASIL. Resolução ํo1/03 - Conselho Nacional de Educação. Câmara de Educação Superior. Institui Diretrizes Curriculares Nacionais dos Cursos de Graduação em Medicina Veterinária. Diário Oficial da 
PFUETZENREITER, M.R.; ZYLBERSZTAJN, A.

União. Brasília, n.37, p.15-6, 20 fev. 2003.

CONSELHO FEDERAL DE MEDICINA VETERINÁRIA. Comissão Nacional de Ensino de Medicina Veterinária. O ensino de medicina veterinária no Brasil. Brasília: CFMV, 1992.

CRUZ, H. M.; ACHA, P. N. La función del médico veterinario en la salud de la comunidad en la América Latina. Educ. Méd. Salud, v.6, n.3-4, p.256-80, 1972.

CUTOLO, L. R. A. Estilo de pensamento em educação médica: um estudo do currículo do curso de graduação em medicina da UFSC. 2001. Tese (Doutorado) - Centro de Ciências da Educação, Universidade Federal de Santa Catarina, Santa Catarina.

FLECK, L. La génesis y el desarrollo de un hecho científico. Madrid: Alianza Editorial, 1986.

PALERMO-NETO, J. Winds of change. Some directions for veterinary medical education as it moves toward the $21^{\text {st }}$ century. Ciênc. Cult., v.47, n.1-2, p.10-2, 1995.

PFUETZENREITER, M. R. 0 ensino da medicina veterinária preventiva e saúde pública nos cursos de medicina veterinária: estudo de caso realizado na Universidade do Estado de Santa Catarina. 2003. Tese (Doutorado) - Programa de Pós-Graduação em Educação, Universidade Federal de Santa Catarina, Santa Catarina.

PFUETZENREITER, M. R.; CUSTÓDIO F. J. F.; KOEPSEL, R. Concepções sobre o conceito de saúde e doença por estudantes de saúde pública. In: ENCONTRO NACIONAL DE PESQUISA EM ENSINO DE CIÊNCIAS, 3., 2001, Atibaia. Atas... Porto Alegre: [s.n.], 2001. 1 cd-rom.

RISTA, A.; BASTOS SANTOS, E. Reuniões da associação de faculdades e escolas de ciências veterinárias do Mercosul: 1999-2001. Associação de faculdades e escolas de ciências veterinárias do Mercosul, 2001.

ROSENBERG, F. J.; OLASCOAGA, R. C. Ciencias veterinarias y sociedad: reflexiones sobre el paradigma profesional. Educ. Méd. Salud, v.25, n.3, p.333-54, 1991.

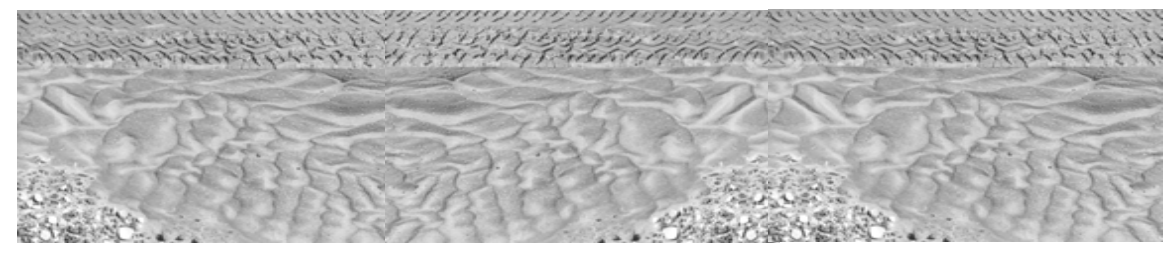

PFUETZENREITER, M.R.; ZYLBERSZTAJN, A. La enseñanza de salud y los curriculos de las carreras de medicina veterinaria: un estudio de caso, Interface - Comunic., Saúde, Educ., $v .8$, n.15, p.349-60, mar/ago 2004.

El trabajo hace un análisis documental de los currículos de las carreras de Medicina Veterinaria pioneras en el país y de la carrera de la Universidad del Estado de Santa Catarina. Se identificaron tres campos de acción principales dentro de la Medicina Veterinaria relacionados con las actividades prácticas realizadas en la profesión, y que corresponden a los contenidos curriculares de las carreras: clínica veterinaria, medicina veterinaria preventiva y salud pública, y zootecnia y producción animal. Fue articulada la relación entre esas áreas y la categoría epistemológica "estilo de pensamiento" propuesta por L. Fleck. El análisis de los datos obtenidos indicó que los conceptos de naturaleza social y preventiva son poco significativos dentro de las carreras de medicina veterinaria, lo cual hace que el estilo de pensamiento de la medicina veterinaria preventiva y salud pública sea poco enfatizado con relación a los otros estilos de pensamiento presentes en la profesión.

PALABRAS CLAVE: Medicina Veterinaria; currículo; educación veterinaria; enseñanza en salud; "estílo del pensamiento".

Recebido para publicação em 20/03/04. Aprovado para publicação em 18/07/04. 